\title{
Size Effect of Light Scattering on the Nano-Sized Color Filter Pigment in Liquid Crystal Display
}

\author{
Chul Gyu Jhun ${ }^{1}$ and Jin Seog Gwag* \\ ${ }^{1}$ School of Green Energy \& Semiconductor Engineering, Hoseo University, Asan 336-795, Korea \\ ${ }^{2}$ Department of Physics, Yeungnam University, Gyeongsan 712-749, Korea
}

(Received December 24, 2013 : revised February 17, 2014 : accepted February 18, 2014)

\begin{abstract}
This study examined the effects of particle size on the light scattering of a nano-sized color filter pigment used to obtain a range of colors in liquid crystal displays. The contrast ratio is one of the most important characteristics of liquid crystal displays. When a color filter is located between two crossed polarizers, the size of the pigment can give rise to a decrease in the contrast ratio due to Rayleigh scattering by the nanoparticles in the filter. The size effect of the color filter pigment on the contrast ratio was investigated in terms of the depolarization parameter. As an experimental result, the depolarization parameter increased with decreasing pigment size. Therefore, a smaller pigment size can reduce light leakage caused by light scattering in the color filter between two crossed polarizers. The depolarization function was also proposed as a useful function for predicting the decrease in the contrast ratio of the color filter.
\end{abstract}

Keywords : Liquid crystal display, Contrast ratio, Color filter, Scattering

OCIS codes : (230.3720) Liquid-crystal devices; (120.2040) Displays; (290.5850) Scattering, Particles

\section{INTRODUCTION}

The contrast ratio (CR) is one of the critical characteristics of display applications. To achieve a high CR, it is important to reduce the light leakage in the black state and increase the transmittance in the white state. Light leakage in the black state of liquid crystal displays (LCDs) is caused by scattering media in the panels. In general, the polarization state of linearly polarized light by an input polarizer is broken slightly at the color filter with nanocomposite media, which can produce light scattering. Owing to partially depolarized light, the output polarizer crossed to the input polarizer cannot block the incident light perfectly. Hence, light leakage occurs, degrading the contrast ratio.

LCDs need to use color filters in each pixel to obtain the desired colors because they are not emissive type displays. On the other hand, most color filters contain nanoparticles that cause light scattering. Figure 1 presents a schematic diagram of the vector expression of the electric field, and light leakage caused by the scattering effect at the color filter when a color filter is sandwiched

\footnotetext{
*Corresponding author: sweat3000@ynu.ac.kr

Color versions of one or more of the figures in this paper are available online.
}

between two crossed polarizers [1-3]. The linearly polarized light is obtained after light passes through the input polarizer, and the oscillation direction of the light is parallel to the transmission axis of the polarizer. When this light meets the nanocomposites in the color filter, light scattering occurs and the polarization state is partially broken. This partially depolarized light becomes randomly polarized states. Of these states, the light component parallel to the transmission axis

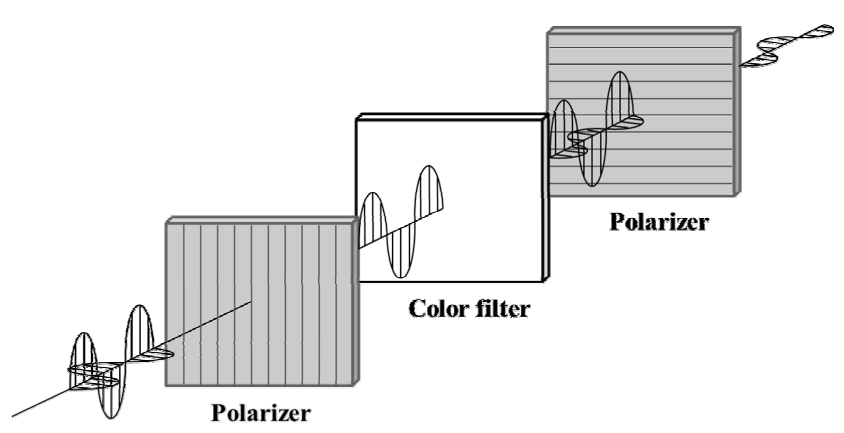

FIG. 1. Schematic diagram of light leakage by the color filter under crossed polarizers. 
of the output polarizer escapes and becomes leakage.

In a more detailed description of the change in polarization, the cause of the polarization change is not for the retardation effects but for light scattering because the retardation value of the color filter is less than a few $\mathrm{nm}$.

This study examined the size effect of a color filter nanocomposite on the contrast ratio in terms of the depolarization parameter.

\section{METHODS}

To examine the scattering effect of nanoparticles in a medium related to depolarization, the size effect of the particle about scattering was analyzed by macroscopic fluctuation theory [4 - 6]. The total scattered power, $P$, is linearly proportional to the incident beam intensity $\mathrm{I}_{0}$ as

$$
P=\sigma I_{0}
$$

where the constant of proportionality is known as the scattering cross section [7]. The scattering cross section, $\sigma$, having the dimensions of area, can be interpreted as the effective geometrical area of the scatter for removing light from the incident intensity.

The optical field was assumed to be of the incident beam intensity $\mathrm{I}_{0}$ as

$$
\vec{E}=E_{0} e^{-i \omega t}
$$

In response to the applied field, the scatter develops a dipole moment

$$
\vec{P}=\varepsilon_{0} \alpha(\omega) \vec{E}
$$

where $\sigma(\omega)$ is the polarizability of the particle. As a consequence of the time-varying dipole moment given by Eq. (3), the particle will radiate. The intensity of radiation at a distance, L, from the scatterer is given by the magnitude of the Poynting vector as follows [7, 8]:

$$
I_{s}=\frac{n\left\langle\ddot{\vec{p}}^{2}\right\rangle}{16 \pi^{2} \varepsilon_{0} c^{3} L^{2}} \sin ^{2} \phi=8 \frac{n \omega^{4} \varepsilon_{0}|\alpha(\omega)|^{2}\left|\vec{E}_{0}\right| 2}{16 \pi^{2} c^{3} L^{2}} \sin ^{2} \phi
$$

A function of the pigment size on the cross-sectional area of scattering can be derived assuming that the scattering particles in the medium are a gathering of dielectric spheres. The dielectric constants of the dielectric sphere and medium are $\varepsilon_{1}$ and $\varepsilon$, respectively. The radius of the dielectric spheres is $a$, and the size of dielectric sphere is much smaller than the wavelength of the light source. The polarizability of the sphere can be expressed as

$$
\alpha=\frac{\varepsilon_{1}-\varepsilon}{\varepsilon_{1}+2 \varepsilon} a^{3}
$$

In addition, the cross-sectional area of scattering can be expressed as Eq. (6). The cross-sectional area of the scattering is proportional to the 6-power of the particle size [7]

$$
\sigma=\frac{8 \pi}{3} \frac{\varpi^{4}}{c^{4}} a^{6}\left(\frac{\varepsilon_{1}-\varepsilon}{\varepsilon+2 \varepsilon_{1}}\right)^{2}
$$

Light leakage is closely related to depolarization. Therefore, the depolarization parameter (D.P.) was used to define the light leakage quantitatively. The depolarization parameter can be expressed as Eq. (7) [9].

Figure 2 shows the measurement method of the depolarization parameter. The white state was used when the

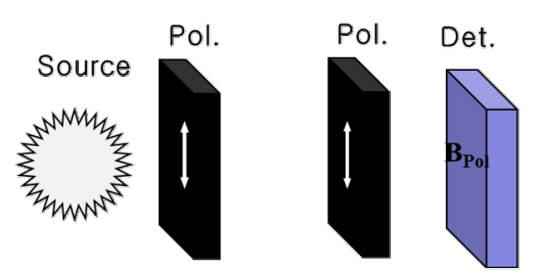

(a)
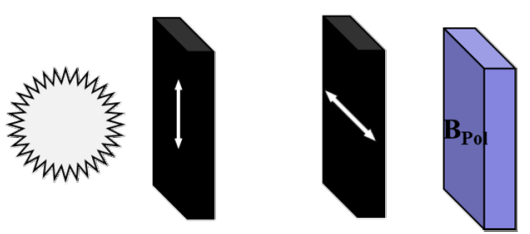

(b)

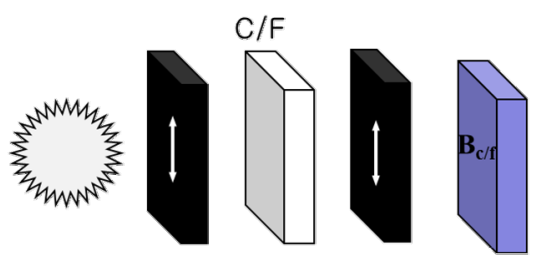

(c)

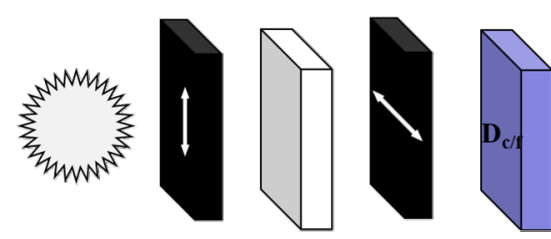

(d)

FIG. 2. Schematic diagram of the depolarization parameter measurement: (a) white state under parallel polarizers, (b) black state under crossed polarizers, (c) white state with color filter between parallel polarizers, and (d) black state with color filter between polarizers. 
transmittance axes of the two polarizers are parallel to each other and the black state when the transmittance axes of the two polarizers are crossed. $C R_{P}$ is the contrast ratio only with the polarizers. The contrast ratio of the polarizers can be calculated as the ratio of the transmittances of the white and black states. A color filter was inserted between the polarizers. In the same manner, the transmittances of the white and black states were measured and the contrast ratio was calculated. $C R_{C F}$ is the contrast ratio when the color filter is located between the two polarizers. Using these values, the depolarization parameter can be obtained as follows

$$
\text { D.P. }=\frac{C R_{C}}{C R_{P}}=\frac{C R_{\text {Cwhite }} / C R_{\text {Cblack }}}{C R_{P \text { white }} / C R_{\text {Pblack }}}
$$

If the light leakage under the crossed polarizers including the color filter is equal to that between the crossed polarizers without a color filter, the depolarization parameter becomes 1. On the other hand, if the depolarization parameter approaches 0 , the light leakage under crossed polarizers including a color filter is increasing compared to the light leakage between crossed polarizers. Therefore, the depolarization parameter can be considered as a factor that represents the reduction ratio of the contrast due to light scattering by the color filter.

\section{RESULTS}

To measure the depolarization parameter with various pigments of the color filter materials, three types of samples were prepared for each red, green and blue pigment. ELS8000 (OTSUKA) was used to measure the mean radius of the pigment by dynamic light scattering. Table 1 lists the measured mean diameters of the pigments.

Various color filter samples made by the various nanosized pigments are located between two polarizers and the transmittance of the samples was measured with CT-1 (TSUBOSAKA) under crossed and parallel polarizers. The contrast ratios of the samples were calculated from measured transmittances, and are listed in Table 2. In two polarizers

TABLE 1. Measured average radius of various pigments for color filter materials

\begin{tabular}{c|c|c|c|c|c|c|c|c|c}
\hline \hline Sample & R 1 & R 2 & R 3 & G 1 & G 2 & G 3 & B 1 & B 2 & B3 \\
\hline Size (nm) & 126 & 85 & 84 & 73 & 58 & 59 & 107 & 52 & 59 \\
\hline
\end{tabular}

without a color filter, the contrast ratio was 100,200:1. The depolarization parameter of the samples, which is represented in Table 2, could then be obtained.

Table 2 also lists the depolarization parameters with various pigment sizes. When the pigment size was 0 , which means no pigment, the depolarization parameter was 1 . The depolarization parameter of the blue color filter with a size of $58 \mathrm{~nm}$ was lower than that of $59 \mathrm{~nm}$. With the exception of this result, the depolarization parameter increased with decreasing pigment size. Therefore, the color filter with a small pigment size can reduce the light leakage caused by the light scattering in the color filter between two crossed polarizers.

\section{DISCUSSION}

From Eq. (2), the scattering cross section of the pigment particle is proportional to the sixth power of the radius. Therefore, the depolarization phenomenon of the pigment particles is proportion to the sixth power of the radius because the depolarization phenomenon is strongly related to the light scattering effects. Therefore, the depolarization parameter can be predicted to be inversely proportional to the sixth power. The depolarization parameter as a function of the size of the pigment particles can be estimated by a depolarization function (D. F.) using the following equation:

$$
\text { D. } \mathrm{F} .=1 /\left(1+\left(\mathrm{x} / \mathrm{x}_{\mathrm{c}}\right)^{6}\right)
$$

where $\mathrm{x}$ is the radius of the scattering particles, which means the particle size of the pigment in the color filter and $\mathrm{x}_{\mathrm{c}}$ is defined as the critical size. In Eq. (8), if the size of the scattering particle is larger or smaller than the critical size, the scattering effects are reduced symmetrically. When the size of the scattering particles is equal to that of the critical size, the depolarization parameter becomes $1 / 2$. Near the critical size, the depolarization parameter is changed dramatically. This was attributed to the scattering effects reaching a relative maximum with the critical size, which is a similar phenomenon of the resonance in the frequency domain.

Using the depolarization function, the depolarization parameter was fitted as a function of the size of the scattering particle, as shown in Fig. 3. From curve fitting, the depolarization parameter began to change over the size of about $20 \mathrm{~nm}$ and it roughly saturated with the size of $100 \mathrm{~nm}$. The resulting critical sizes for the red, green and blue color filter were $74 \mathrm{~nm}, 55 \mathrm{~nm}$ and $40 \mathrm{~nm}$, respectively.

TABLE 2. Measured contrast ratio and calculated depolarization parameters of the various color filter samples

\begin{tabular}{c|c|c|c|c|c|c|c|c|c|c}
\hline \hline Sample & Air & R 1 & R 2 & R 3 & G 1 & G 2 & G 3 & B 1 & B 2 & B3 \\
\hline CR & 100,200 & 3,000 & 23,000 & 39,000 & 13,200 & 38,400 & 44,600 & 9,800 & 14,300 & 15,800 \\
\hline Dep. Parameter & & 0.03 & 0.23 & 0.39 & 0.13 & 0.38 & 0.45 & 0.1 & 0.14 & 0.16 \\
\hline
\end{tabular}




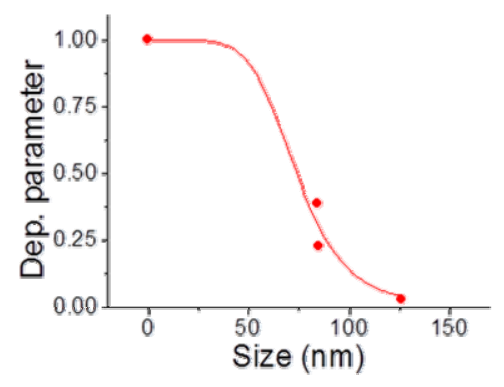

(a)

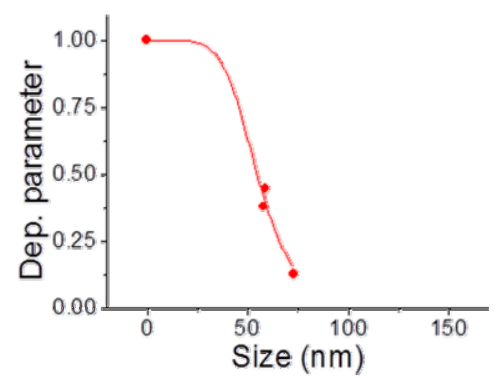

(b)

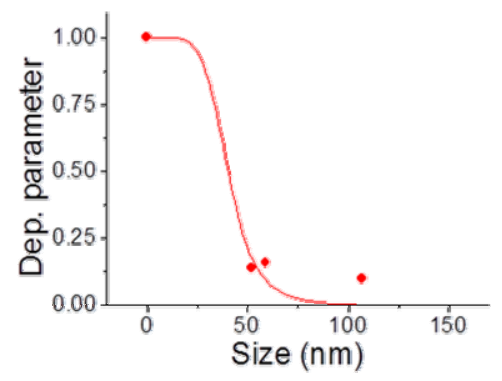

(c)

FIG. 3. Curve fitting by depolarization function for (a) red, (b) green, and (c) blue color filters.

This shows that the critical size is approximately 0.1 times the wavelength of the transmitted light of each color filter.

Rayleigh scattering is mostly associated with light scattering by small particles, in which small refers to the size parameter $x<<1$, with $x=2 \pi r / \lambda[10]$. H. Moosmüller calculated various scattering effects as function of the particle size parameter. By the single scatter with refractive index of 1.55, Rayleigh scattering occurs when the size parameter is between 0.1 and 1.2. For the wavelength of $550 \mathrm{~nm}$, the size parameter $\mathrm{x}$ can be converted into the particle size $\mathrm{r}$, which is between $8.75 \mathrm{~nm}$ and $105 \mathrm{~nm}$. This Rayleigh scattering regime agrees with our fitting function.

Consequently, to reduce the light leakage in liquid crystal displays, the size of the nano-particles in the color filter should be much smaller than the critical sizes for each wavelength. Such applications are expected to produce high quality LCDs with the optimal contrast ratio.

\section{CONCLUSION}

The contrast ratio is one of the most important charac- teristics of liquid crystal displays. The color filter in a liquid crystal panel is commonly used. When the color filter is located between two crossed polarizers, the size of the pigment can give rise to a decrease in the contrast ratio due to Rayleigh scattering at the nanoparticles in the filter. This study examined the size effect of the color filter pigment on the contrast ratio in terms of the depolarization parameter. The depolarization function, which is a useful function to predict the reduction of the contrast ratio of the color filter, was also proposed. As a result, to increase the contrast ratio in liquid crystal displays, the size of the nanoparticles in the color filter should be much smaller than the critical sizes for each wavelength.

\section{ACKNOWLEDGMENT}

This study was supported by a grant from Dongwoo Fine-Chem in 2007 and the Human Resources Development Program (No. 20124030200100) of the Korea Institute of Energy Technology Evaluation and Planning (KETEP) grant funded by the Korea government Ministry of Trade, Industry and Energy.

\section{REFERENCES}

1. Y. Utsumi, I. Hiyama, Y. Tomioka, K. Kondo, and S. Matsuyama, "Analysis of light leakage caused by color filter between crossed polarizers," Jpn. J. Appl. Phys. 46, 1047-1050 (2007).

2. M. Yoneya, Y. Utsumi, and Y. Umeda, "Depolarized light scattering from liquid crystals as a factor for black level light leakage in liquid-crystal displays," J. Appl. Phys. 98, 016106-016106-3 (2005).

3. Y. Utsumi, I. Hiyama, Y. Tomioka, and K. Ono, "Quantitative analysis method for measuring light leakage intensity of three primary color filters placed between crossed polarizers,” Jpn. J. Appl. Phys. 47, 3518-3521 (2008).

4. A. Einstein, "The theory of the opalescence of homogeneous fluids and liquid mixtures near the critical state," Ann. Phys. 33, 1275-1298 (1910).

5. L. D. Landau, E. M. Lifshitz, and L. P. Pitaevskii, Electrodynamics of Continuous Media, 2nd ed. (ButterworthHeinemann, Burlington, MA, USA, 1984).

6. G. A. Miller, "Fluctuation theory of the resonance enhancement of Rayleigh scattering in absorbing media," J. Phys. Chem. 82, 616-618 (1978).

7. R. W. Boyd, Nonlinear Optics, 3rd ed. (Academic Press, Burlington, MA, USA, 2008).

8. J. D. Jackson, Classical Electrodynamics, 3rd ed. (John Wiley \& Sons, Inc., New York, NY, USA, 1998).

9. T. Koseki, T. Kukunaga, H. Yamanaka, and T. Ueki, "Color filter for 10.4-in.-diagonal 4096-color thin-film-transistor liquid crystal displays,” IBM J. Res. Develop. 36, $43-50$ (1992).

10. H. Moosmüller and W. P. Arnott, "Particle optics in the Rayleigh regime," J. Air \& Waste Manage. Assoc. 59, 1028-1031 (2009). 